\title{
ESTIMAÇÃO DE PARÂMETROS GENÉTICOS ENTRE PESOS PRÉ E PÓS-DESMAMA NA RAÇA NELORE
}

\author{
GENETIC PARAMETERS ESTIMATION AMONG PRE AND POST WEANING WEIGHTS IN \\ NELLORE BREED
}

\author{
Garnero, A.V. ${ }^{1 A}$, Muñoz, M.C.C.D. ${ }^{2}$, Marcondes, C.R. ${ }^{3}$, Lôbo, R.B. ${ }^{4}$, Lira, T. ${ }^{5}$ e Gunski, R.J. ${ }^{1 *}$ \\ ${ }^{1}$ Campus São Gabriel. UNIPAMPA. Av Antônio Trilha, n 1847. CEP 97300-000. São Gabriel, RS. Brasil. \\ Aanaliagarnero@yahoo.com.br; *rgunski@yahoo.com.br \\ ${ }^{2}$ Estación Experimental Agropecuaria. INTA Cerro Azul. Ruta Nacional nº 14, km 836. CP 3313. Misiones. \\ Argentina. martind@cerro.inta.gov.ar \\ ${ }^{3}$ Empresa Brasileira de Pesquisa Agropecuária. Amazônia Oriental. Tv. Dr. Enéas Pinheiro, s/n Marco. CEP \\ 66095-100. Belem, PA. Brasil. cimarcon@yahoo.com \\ ${ }^{4}$ Associação Nacional de Criadores e Pesquisadores, ANCP. Ribeirão Preto-SP. CEP 14020-230. Brasil. \\ raysildo@ancp.org.br \\ ${ }^{5}$ Instituto Nacional de Pesquisa da Amazônia, INPA. Laboratório de Genética Animal - CPBA. Av André \\ Araújo, 2936. Petrópolis. CEP 69083-000. Manaus, AM. Brasil.
}

\section{PaLAVRAS CHAVE ADICIONAIS \\ Bovinos. Covariância. Herdabilidade.}

\section{RESUMO}

As correlações entre duas ou mais características estimam o nível de união entre elas, sendo a origem e a grandeza da relação existente de grande importância, pois visam aprimorar o material genético de um conjunto de caracteres que agem simultaneamente. Os objetivos foram: estimar os componentes de variância e herdabilidade para os pesos padronizados aos $120,240,365$, 450 e 550 dias de idade (P120, P240, P365, P450 e P550, respectivamente) e estimar a correlação genética entre o P120 e os outros pesos citados. Foram utilizadas 29769 observações de bovinos da Raça Nelore, em análises uni e bi-caráter. Estas últimas, com o P120 como característica principal e a covariância aditiva fixada, estrategicamente, com o intuito de se obter estimativas de correlações genéticas. Os valores estimados de herdabilidades foram: 0,21 (P240); 0,37 (P365); 0,36 (P450) e 0,42 (P550). Para o P120, as estimativas variaram de 0,23 a 0,31 . Os valores das correlações genéticas entre o $\mathrm{P} 120$ e os outros pesos foram altos e positivos: 0,96 (P240); 0,87 (P365); 0,79 (P450) e 0,76 (P550), sugerindo que a seleção para pesos em idade mais jovens promoverá o aumento dos pesos posteriores.

\author{
AdDITIONAL KEYWORDS \\ Bovines. Covariance. Heritability.
}

\section{SUMMARY}

Correlations among two or more traits indicate the extent to which they are associated. The level and magnitude of this association are key factors in genetic improvement because the goal is to enhance the genetic makeup of a group of traits that act simultaneously. Therefore, the aim of this study was to estimate the components of variance and heritability for standard weights at 120, 240, 365,450 and 550 days of age (P120, P240, P365, $\mathrm{P} 450$ and P550, respectively), and the genetic correlation among P120 and other weights in Nellore cattle using a database of 29769 records. Bivariate analysis was carried out for all the traits using P120 as an anchor, and additive covariance was fixed as an analytical strategy in order to obtain genetic correlation estimates. Heritability $\left(h^{2}\right)$ values were 0.21 for $P 240 ; 0.37$ for $P 365 ; 0.36$ for P450 and 0.42 for P550. The estimation of $h^{2}$ varied between 0.23 and 0.31 for W120. Genetic correlation estimates were high and positive, 0.96 , $0.87,0.79$ and 0.76 among P120 and P240, P365, $P 450$ and P550, respectively. These values indicate that selection for weight at younger ages will lead to a corresponding change in later weight, in the same direction.

Arch. Zootec. 59 (226): 307-310. 2010. 


\section{INTRODUÇÃO}

Os parâmetros genéticos constituem a ferramenta utilizada na obtenção de predições das respostas direta e correlacionada à seleção, do valor genético dos animais e na elaboração de índices de seleção (Lira et al., 2008). A herdabilidade de um caráter, em uma população, é o parâmetro que irá determinar a estratégia a ser usada em seu melhoramento, pois mede a capacidade de transmissão desse caráter à sua progênie. Por sua vez, a correlação permite predizer como uma característica deverá se comportar, a partir de parâmetros obtidos para outra e estima o nível de união entre elas.

Os objetivos deste trabalho foram estimar os componentes de variância e herdabilidade para os pesos padronizados aos $120,240,365,450$ e 550 dias de idade e a correlação genética entre o P120 e os outros pesos citados, em bovinos da Raça Nelore.

\section{MATERIALE MÉTODOS}

Foram analisadas 29769 observações de bovinos Nelore do Brasil. A matriz de parentesco totalizou 46310 animais e, as características estudadas foram os pesos padronizados aos 120, 240, 365, 450 e 550 dias de idade(P120, P240, P365, P450 e P550, respectivamente).

As análises estatísticas básicas e a preparação dos arquivos foram realizadas utilizando o software Statistical Analysis System (SAS, 1996) e a estimação dos parâmetros genéticos, pelo Método dos Modelos Mistos sob Modelo Animal utilizando o software Multiple Traits Derivative Free Restricted Maximum Likelihood (Boldman et al., 1995).

Foram realizadas análises uni e bicaráter, sendo estas últimas, entre o P120 e os demais pesos estudados. Para o estudo das características pré-desmama (P120 e P240), utilizou-se o modelo 1, que incluiu como efeitos fixos, os grupos de contem- porâneos e classes de idade da vaca ao parto, e como aleatórios, os efeitos genéticos direto, materno e de ambiente permanente da vaca, além do residual. Para as características pós-desmama, utilizou-se o modelo 2, que incluiu como fixos, os efeitos de grupo de contemporâneos e classes de idade da vaca ao parto e, como aleatórios, os efeitos genéticos direto e residual.

Em termos matriciais os modelos podem ser descritos como:

$$
\begin{aligned}
& Y=X \beta+Z_{1} a+Z_{2} m+Z_{3} p+\varepsilon(\text { modelo } 1) \\
& Y=X \beta+Z_{1 a}+\varepsilon(\text { modelo } 2)
\end{aligned}
$$

em que:

$Y=$ vetor das observações de cada característica;

$X=$ matriz de incidência dos efeitos fixos;

$\beta=$ vetor dos efeitos fixos;

$Z_{1}=$ matriz de incidência do efeito genético direto de cada animal;

$\mathrm{a}=$ vetor de efeitos aleatórios genéticos diretos;

$Z_{2}=$ matriz de incidência do efeito genético materno de cada animal;

$m=$ vetor de efeitos aleatórios genéticos maternos;

$Z_{3}=$ matriz de incidência do efeito de ambiente permanente;

$p=$ vetor de efeitos aleatórios de ambiente permanente;

$\boldsymbol{\varepsilon}=$ vetor de efeitos aleatórios residuais.

Os grupos de contemporâneos (GC) foram constituídos por animais nascidos na mesma fazenda e época do ano e com o mesmo sexo e regime alimentar (pasto ou suplementação). Para a formação dos grupos de contemporâneos aos 120 e 240 dias (GC120 e GC240) foram considerados quatro trimestres de nascimentos (de janeiro a março, de abril a junho, de julho a setembro e de outubro a dezembro), totalizando $451 \mathrm{e}$ 554 grupos diferentes, respectivamente. Enquanto que, para os grupos de contemporâneos aos 365, 450 e 550 dias (GC365, GC450 e GC550) utilizaram-se semestres de nascimento: de janeiro a junho e de julho a dezembro, totalizando 544, 578 e 564 grupos diferentes, respectivamente. A idade da vaca 
Tabela I. Número de observações (N), média, coeficiente de variação (CV), máximo e mínimo em $\mathrm{kg}$ dos pesos padronizados $(P)$ aos 120, 240, 365, 450 e 550 dias de idade. (Number of observations $(\mathrm{N})$, average, coefficient of variation $(\mathrm{CV})$, maximum and minimum in $\mathrm{kg}$ of standardized weights $(P)$ in 120, 240, 365, 450 and 550 days of age)

\begin{tabular}{lccccc}
\hline Peso dias & $N$ & Média & CV\% & Máximo & Mínimo \\
\hline P120 & 21799 & 129 & 15,64 & 155 & 85 \\
P240 & 22902 & 244 & 7,87 & 275 & 205 \\
P365 & 21646 & 365 & 7,50 & 410 & 320 \\
P450 & 19617 & 452 & 5,98 & 500 & 410 \\
P550 & 16169 & 552 & 5,00 & 595 & 505 \\
\hline
\end{tabular}

ao parto foi agrupada em seis classes etárias distintas: menor que 36 , de 36 a 47 , de 48 a 59 , de 60 a 71, de 72 a 119 , e maior/igual a 120 meses de idade.

Considerou-se que a convergência foi atingida, quando a variância dos valores da função $(-2 \log \mathrm{L})$ fosse menor que $10^{-9} \mathrm{e}$, a cada convergência o programa foi reiniciado (entre 3 a 5 vezes, de acordo as características), usando como valores de (co)variâncias iniciais aqueles obtidos na análise anterior (valores fixos), checando assim, possíveis máximos locais, assegurando que o valor alcançado fosse o máximo global e facilitando a obtenção das correlações genéticas.

\section{RESULTADOSEDISCUSSÃO}

As médias dos pesos padronizados são apresentadas na tabela I e estão em consonância com as reportadas pela literatura. As estimativas de herdabilidade genética direta para os pesos padronizados aos 120 e 240 dias de idade (P120 e P240) foram de mediana magnitude $(0,23$ e 0,21 , respectivamente) (tabela II), correspondentes aos valores encontrados na literatura (Lira etal., 2008).

O coeficiente de herdabilidade materna para $\mathrm{P} 120$ variou de 0,04 a 0,09 , indicando a grande importância do ambiente de criação proporcionado pela matriz ao bezerro, especialmente nesta idade, que corresponde ao pico da curva de lactação em zebuínos. O P120 é utilizado, inclusive, na avaliação da habilidade maternal e do crescimento prédesmama do bezerro, sendo uma das mais usada como característica-base, em análises bi-caráter, por minimizar os efeitos de descartes posteriores à desmama. A estimativa de herdabilidade materna para o P240, foi de

Tabela II. Componentes de (co)variância e valores de herdabilidade provenientes das análises bi-caráter entre o peso $(P)$ padronizado aos 120 dias e os 240, 365, 450 e 550 dias de idade. (Components of (co) variance and values of heritability proceeding from analyses two-traits among the weight $(\mathrm{P})$ standardized in 120 days and in $240,365,450$ e 550 days of age).

\begin{tabular}{|c|c|c|c|c|}
\hline & \multicolumn{4}{|c|}{ P120(1) } \\
\hline & P240(2) & P365(2) & $P 450(2)$ & P550(2) \\
\hline$\sigma_{a 1}^{2}$ & 49,00 & 70,53 & 55,42 & 62,82 \\
\hline & 65,00 & 108,90 & 90,00 & 116,00 \\
\hline & 94,01 & 220,85 & 235,62 & 369,47 \\
\hline & 10,34 & 8,67 & 11,60 & 13,96 \\
\hline$\sigma^{-}$ & 19,76 & - & - & - \\
\hline$\sigma_{t}$ & 121,00 & 116,77 & 126,96 & 124,40 \\
\hline$\sigma^{2}$ & 265,70 & 373,39 & 424,35 & 509,07 \\
\hline$\sigma^{2}$ & 215,13 & 226,80 & 229,83 & 238,01 \\
\hline 0 & 437,41 & 594,32 & 659,97 & 878,55 \\
\hline & 0,23 & 0,31 & 0,24 & 0,26 \\
\hline $\mathrm{n}^{2}$ & 0,21 & 0,37 & 0,36 & 0,42 \\
\hline & 0,09 & 0,04 & 0,05 & 0,06 \\
\hline $\mathrm{h}^{2}{ }_{\mathrm{m} 2}$ & 0,05 & - & - & - \\
\hline$r_{g_{1,2}}$ & 0,96 & 0,87 & 0,79 & 0,76 \\
\hline $\mathrm{c}^{2}{ }_{1}$ & 0,16 & 0,13 & 0,15 & 0,15 \\
\hline $\mathrm{C}^{2}{ }_{2}$ & 0,13 & - & - & - \\
\hline $\mathrm{e}^{2}{ }_{1}$ & 0,56 & 0,51 & 0,55 & 0,52 \\
\hline$\Omega^{2}$ & 0,61 & 0,63 & 0,64 & 0,58 \\
\hline
\end{tabular}

$\sigma_{a}^{2}$ : variância genética direta, $\sigma_{a 1,2}^{2}$ : covariância genética aditiva, $\sigma_{m}^{2}$ :variância genética materna, $\sigma_{p}^{2}$ : variância fenotípica, $\sigma_{e}^{2}$ : variância residual, $h_{a}^{2}$ : herdabilidade direta, $\mathrm{h}_{\mathrm{m}}{ }_{\mathrm{m}}$ : herdabilidade materna, $r_{\mathrm{g}}$ : correlação genética, $c^{2}$ : contribuição do ambiente permanente para a variância fenotípica total, $e^{2}$ : fração do erro residual. 


\section{GARNERO, MUÑOZ, MARCONDES, LÔBO, LIRA E GUNSKI}

0,05 concordando com Siqueira et al. (2003) e Lira et al. (2008). Para o peso ao ano (P365), característica que expressa a habilidade do animal em ganhar peso no período pósdesmama, o coeficiente de herdabilidade direta estimado $(0,37)$ foi ligeiramente superior àqueles publicados na literatura (Lira et al., 2008).

Os pesos padronizados aos 450 e 550 dias de idade (P450 e P550), expressam a habilidade do animal em ganhar peso no período pós-desmama. Existe tendência de redução da idade do peso ao sobreano, com a finalidade de intensificar o processo de seleção, procurando selecionar animais que atinjam pesos desejados com a maior precocidade produtiva possível. A herdabilidade genética direta estimada para P450 foi inferior à reportada por Siqueira et al. (2003), utilizando o mesmo modelo de análise, e semelhante aos valores relatados no artigo de revisão de Lira et al. (2008). O valor estimado de herdabilidade sugere que essa característica pode promover ganhos genéticos apreciáveis, mediante seleção.

Em relação ao ambiente permanente da vaca para as características P120 e P240, variaram de 0,13 a 0,16 . Esta parcela da variação fenotípica é descrita como derivada da vaca, em virtude de fatores que podem provocar diferenças permanentes entre as matrizes de um rebanho.

As correlações obtidas (tabela II) permitem inferir que a seleção para qualquer

\section{BIBLIOGRAFIA}

Boldman, K.G., Kriese L.A., Van Vleck, L.D., Van Tasssel, C.P. and Kachman, S.D. 1995. A manual for use of MTDFREML: a set of programs to obtain estimates of variance and covariance [DRAFT]. Agricul. Res. Service. Lincoln. 120 pp.

Lira, T., Rosa, E.M. e Garnero, A.V. 2008. Parâmetros genéticos de características produtivas e reprodutivas em zebuínos de corte um dos pesos deverá promover mudanças positivas nos outros. Em relação a este parâmetro, há uma discussão recorrente no que diz respeito à obtenção de estimativas que utilizem em seu cálculo medições do peso aos 550 dias de idade. Para que este processo ocorra faz-se necessária a permanência do animal no rebanho por, pelo menos, três meses a mais do que seus 450 dias de idade, demandando com isso, mais gastos por parte do criador para a manutenção desse animal. A correlação genética entre o P450 e o P120 foi maior do que a entre este último e o P550, demonstrando, assim que é perfeitamente possível a redução do número de pesagens e a seleção dos animais em idades mais jovens.

\section{CONCLUSÕES}

Através da análise das estimativas de herdabilidades e correlações obtidas podese concluir que há variabilidade genética nos rebanhos analisados, podendo ser as características estudadas usadas em um processo de seleção. Esta seleção, objetivando o aumento dos pesos a idades mais jovens, promoverá, também, mudanças favoráveis nos pesos a idades posteriores.

\section{AGRADECIMENTOS}

Os autores agradecem ao apoio financeiro do CNPq, PRONEX, FAPESP,FINEP,ANCPe criadores do PMGRN, CNPq/SEPLAN-TO.

(Revisão). Ciência Animal Bras., 9: 1-22.

SAS. 1996. Statistical Analysis System. 6.12 versão for Windows ${ }^{\circledR}$. SAS Institute. Cary.

Siqueira, R.L.P.G., Oliveira, J.A., Lôbo, R.B., Bezerra, L.A.F. e Tonhati, H. 2003. Análise da variabilidade genética aditiva de características de crescimento na raça Nelore. Rev. Bras. Zootecn., 32: 99-105. 